\title{
Intercostal Artery Reattachment Guided by Intraoperative Neurophysiologic Monitoring During Open Thoracoabdominal Aortic Repair: Basic Principles and Clinical Experience
}

\author{
Ubaldo Del Carro, Francesca Bianchi, Marco Cursi, \\ and Heike Caravati
}

Spinal cord ischemia (SCI) is one of the most important complications during thoracoabdominal aortic aneurysm (TAAA) repair. In an effort to reduce this risk, over the last few years, operative strategies have evolved from the traditional clamp-and-sew technique with epidural cooling to one applying distal aortic perfusion with a variety of additional strategies to protect the spinal cord [1]. Among these strategies, intraoperative neuromonitoring (IOM) allows for a real-time assessment of spinal cord functional integrity and early detection of SCI, providing the team with the opportunity to promptly trigger interventions for maximizing spinal perfusion and potentially reverse spinal injury [2-4]. Type I to III TAAAs are often repaired with distal perfusion techniques and neuromonitoring, while the use of such adjuncts is irregularly applied in the repair of type IV TAAA [5-7]. Literature data support the use of

\footnotetext{
U. Del Carro $(\bowtie)$

Department of Neurology and INSPE, Vita-Salute University, San Raffaele Scientific Institute,

Milan, Italy

e-mail: delcarro.ubaldo@hsr.it

F. Bianchi - M. Cursi · H. Caravati

Department of Neurology and INSPE, San Raffaele

Scientific Institute, Milan, Italy

e-mail: bianchi.francesca@hsr.it;

cursi.marco@hsr.it; caravati.heike@hsr.it
}

IOM as a strategy to detect SCI and to guide hemodynamic optimization and/or reimplantation of intercostal arteries to prevent or treat SCI, providing a level $\mathrm{B}$ of evidence [8]. The neuromonitoring modalities used during TAAA surgery are the somatosensory evoked potentials (SSEPs) and the motor evoked potentials (MEPs) because their neural pathways pass through the spinal cord and their sensitivity to ischemia [9]. These techniques are safe, do not interfere with surgical procedures, or modify the clamping time. SSEPs provide information about the somatosensory pathway in the spinal dorsal columns. Furthermore, their peripheral response (i.e., popliteal potential) is useful to monitor the impending peripheral nerve injury and therefore differentiates SCI from peripheral ischemia. Nielsen and Kardel studied the peripheral nerve conduction during ischemia and found that sensory conduction velocity exhibits a temporospatial slowing along the ischemic nerve segment, determining that conduction time increases and amplitude decreases rectilinearly with the squared duration of ischemia and proportionally to segment length [10]. Because TAAA surgery is more likely to compromise blood flow in the anterior spinal artery territory, the presence of synapses in the anterior horn gray matter makes MEPs more sensitive than SSEPs in detecting SCI. Furthermore, MEP monitoring has a better 
correlation with motor outcome because it monitors the corticospinal tract [9]. However, SSEPs can provide a neurophysiologic "background" on which to interpret MEP changes, and the use of both these techniques may offer a better vigilance on neurological complication [9]. MEPs are elicited by transcranial electrical stimulation applied through corkscrew electrodes placed at FC3 and FC4 scalp sites and recorded from limb muscles. The electrical stimulation consisted in a train-offive monophasic or biphasic stimuli of $500 \mu \mathrm{s}$ duration, intensity ranging from 200 to $400 \mathrm{~V}$ (avoiding to exceed $600 \mathrm{~V}$ ), and interstimulus interval of $4 \mathrm{~ms}$. MEPs are recorded by needle electrodes placed into limb muscles and monitored bilaterally in lower limbs. Responses from the upper limb are frequently used as internal control to rule out the effects of systemic factors. There is no literature agreement on the MEP warning criteria, and even if different percentages of amplitude reduction compared to the baseline value have been proposed, a recent meta-analysis showed that the "all-or-none" cutoff may be the best criterion [11]. SSEPs are obtained by electric stimulation of the posterior tibial nerve at the ankle and usually recorded from the popliteal fossa and the scalp. The right and left posterior tibial nerves are alternatively stimulated at the internal malleolus. The stimulus consists in a single current pulse, with intensity high enough to obtain motor twitch, duration of $200 \mu$ s, and repetition rate of $4.6 \mathrm{~Hz}$. Cortical potentials are recorded by means of corkscrew electrodes using a multichannel montage that includes $\mathrm{Fz}-\mathrm{CPz}$ and CP3-CP4 derivations. Peripheral potentials are recorded bilaterally by needle electrodes placed in the popliteal fossa. A range of 300-500 repetitions are averaged to produce reproducible SSEPs. The traditional 10/50 rule is considered to define SSEP abnormalities (Fig. 26.1). A baseline MEP
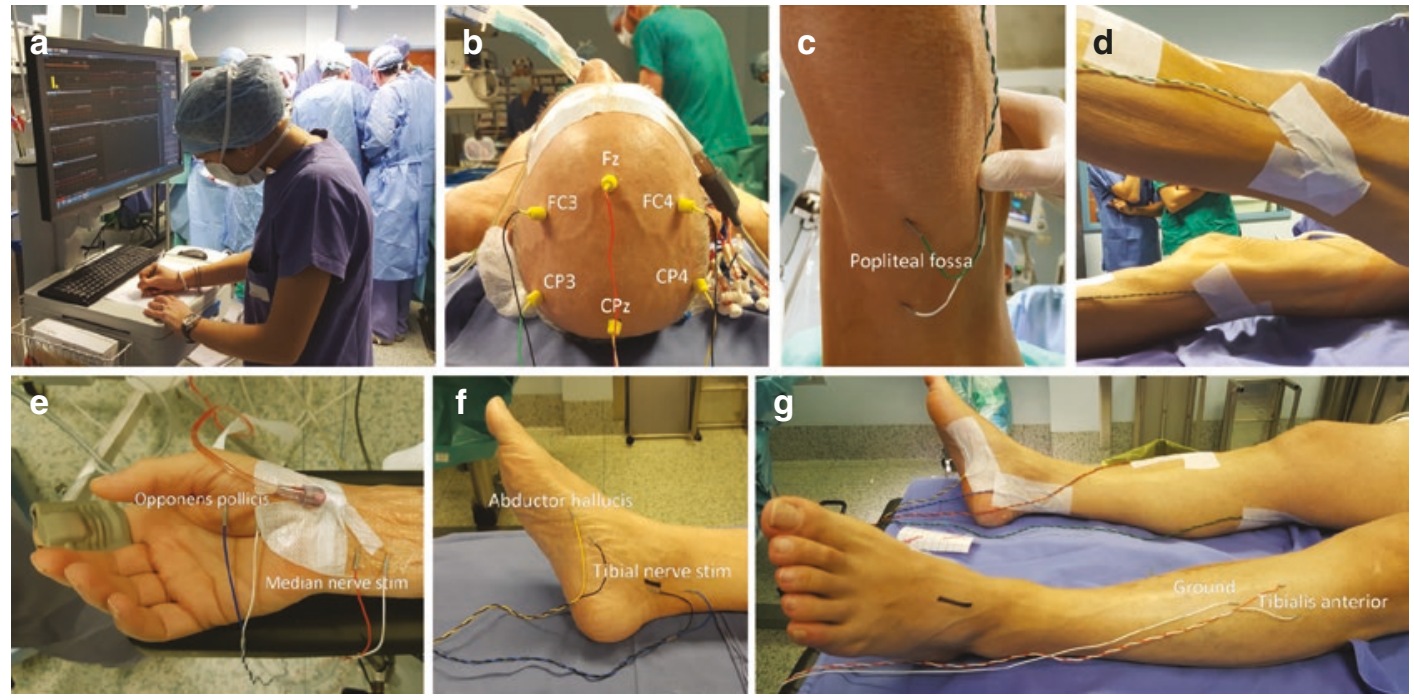

Fig. 26.1 Neurophysiological intraoperative monitoring during TAAA repair. The neurophysiological monitoring system is located in the operating room near the surgical table (a). Corkscrew electrodes are used to record SSEPs and to deliver the transcranial electrical stimulation for MEPs (b). Cortical SSEPs are recorded from two channels: $\mathrm{Fz}-\mathrm{CPz}$ and $\mathrm{CP} 3-\mathrm{CP} 4$. The transcranial electrical stimulus for MEPs is applied between FC3 and FC4. Twisted pair needle electrodes are placed at the left (c) and right popliteal fossa to record SAPs. Needle electrodes are secured to the patient skin with Hypafix non-woven fabric adhesive patch $(\mathbf{d}, \mathbf{g})$. Twisted pair needle electrodes are placed on the right opponens pollicis muscle for recording three different responses: the MEP, the MAP, and the TOF. Another couple of needle electrodes is placed at the wrist to stimulate the median nerve for obtaining TOF and MAP responses (e). Twisted pairs needle electrodes are positioned bilaterally on the abductor hallucis (f) and tibialis anterior (g) for recording lower limb MEPs. Two couples of twisted needle electrodes are placed bilaterally at the ankle (f, g) to stimulate the tibial nerve for obtaining MAPs and SSEPs. The ground electrode is placed on the left leg (g) 
and SSEP recording is suggested after anesthetic induction and intubation maneuvers, in order to guarantee the measurements when a steady anesthetic state has been reached and the effect of the initial dose of muscle relaxant has disappeared. All subsequent measurements are compared with the baseline recordings. MEPs are checked intermittently until the aorta is cross-clamped and more regularly during and after the crossclamping (e.g., every $5 \mathrm{~min}$ ). SSEPs are monitored continuously. A critical aspect of this neurophysiologic monitoring is the interpretation of the MEP and SSEP changes occurring after aortic cross-clamping, specifically the distinction between those changes due to SCI and falsepositive results. There is a clear need to better interpret these changes in order to guide more precisely the choice of appropriate surgical strategies for each patient, increasing the speed and safety of the procedures and avoiding unnecessary time-consuming maneuvers. Intraoperative evoked potential (EP) changes may result from ischemia of their specific neural pathway or may be due to nonspecific systemic factors. When systemic factors take place, there is a concomitant reduction of both upper and lower limb EPs. Hence, the simultaneous use of an upper limb recording represents an important internal control to detect unspecific EP changes. Among systemic causes that may influence EPs, there are physiologic factors (e.g., blood pressure, temperature) and pharmacologic agents (i.e., anesthetic drugs, neuromuscular blocking agents). The use of inhalation anesthetic agents has an inhibitory effect on neurotransmission, and total intravenous anesthesia is widely recommended when neurophysiological monitoring is needed. Deepening anesthesia and administering boluses decrease EP responses, and the maintenance of a stable concentration of anesthetics is advisable. Several different methods of EEG processing have been developed to evaluate the intraoperative anesthetic depth, and among these the bispectral index (BIS) provides a quantitative measure easy to interpret. Neuromuscular blockade can influence IOM results, especially decreasing MEP signals. For this reason, it is often omitted after intubation or controlled by means of different methods throughout the surgery. Among these, the train-offour (TOF) test is based on four supramaximal stimulation of peripheral nerve resulting in four twitches: T1, T2, T3, and T4. The assessment of the neuromuscular blockade is performed by measurement of the amplitude of the compound muscle action potential (CMAP) and calculation of percentage of CMAP decrement from T1 to T4. Hypothermia decreases the reliability of EP monitoring, even if a stable temperature above $32{ }^{\circ} \mathrm{C}$ seems to be sufficient enough to record the signal. Once excluding systemic factors, the role of hypoperfusion must be considered. Hypoperfusion can be due to both surgical maneuvers and arterial hypotension and can involve the spinal cord and/ or lower limbs. In both of these cases, an alteration of lower limb EPs with preserved upper limb response should be expected. Lower limb ischemia may occur when the surgeon performs distal aortic or iliac anastomosis and suspends retrograde perfusion, or it happens frequently in the left leg used for femoral artery cannulation. To overcome this limitation, several studies proposed monitoring only the right leg, others the use of a left femoral side graft rather than a second cannula to maintain the left leg perfused and available for monitoring cord functions. SSEPs could be useful to identify limb ischemia, showing a concomitant alteration of popliteal and cortical potentials. Another method consists in the study of CMAP is obtained by stimulating a peripheral nerve (i.e., tibial nerve at the ankle) and recording the motor response from the muscle (i.e., abductor hallucis). CMAP provides a further intraoperative measure of the peripheral nerve functional integrity and could be useful to differentiate SCI from limb ischemia. When CMAPs and MEPs are both reduced at the lower limbs, the surgeon can suspect that EP changes are due to peripheral rather than spinal ischemia. In such scenario, more complexed maneuvers, such as selective perfusion or reimplantation of intercostal arteries, may be unnecessary. On the other hand, when MEPs are altered and CMAPs remain unchanged, the surgeon is sufficiently confident that an ischemic injury to the spinal cord is ongoing. 


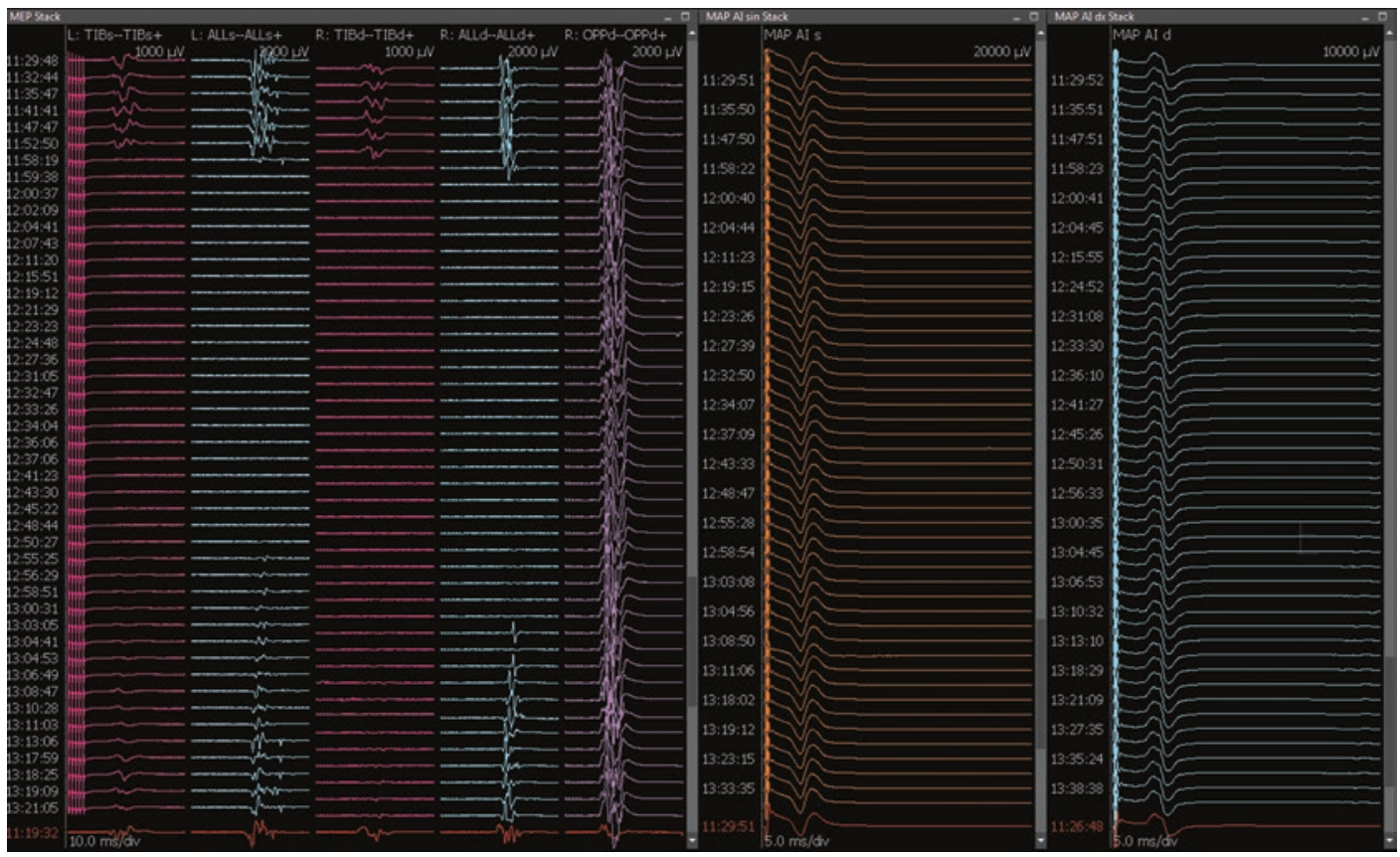

Fig. 26.2 Case 1: spinal cord ischemia after aorta crossclamping. In the figure MEP and CMAP time courses are reported for patient (AM) with TAAA type II. From left to right: MEPs recorded from the left tibialis anterior (first column), left abductor hallucis (second column), right tibialis anterior (third column), right abductor hallucis (fourth column), and right opponens pollicis (fifth column) and CMAPs recorded from left and right abductor hallucis (sixth and seventh columns). Baseline tracings (represented by the red line) are shown at the bottom of

A selection of clinical cases is reported in Figs. 26.2, 26.3, 26.4, 26.5, 26.6, and 26.7. In order to verify the sensitivity of CMAP amplitude in peripheral ischemia and the usefulness of this method combined with MEP and SSEP monitoring in differentiating spinal versus peripheral ischemia, we draw a prospective open-label study on patients with TAAA undergoing surgical repair. The study has been approved by local ethics committee on 8 October 2015 and registered on ClinicalTrials.gov website (ID: NCT02644681), and it is still ongoing. The segmental artery reimplantation represents a strategy to reduce the risk of SCI based on specific pathophysiologic prerequisite, especially when the other strategies are not able to restore MEP changes. The pathophysiology of spinal cord injury in TAAA surgery is essentially based on ischemia-infarction model. Thoracic aortic the stack. Aorta cross-clamping occurred at 11:32. At 11:58 MEPs were abruptly lost in the left lower limb and, $1 \mathrm{~min}$ later, also in the right lower limb. No changes were observed in upper limb MEPs and in lower limb CMAPS. Distal aortic clamp was removed, and anterograde lower extremity perfusion restored at 12:22 but MEPs did not recover. Six intercostal arteries were identified and reattached to the graft, determining a slow restoration of MEPs to the baseline levels

clamping reduces arterial blood supply to the spinal cord, increases spinal fluid pressure, and produces inadequate oxygen delivery and ischemia [12-14]. Infarction occurs when the ischemic tolerance of the spinal cord is exceeded or when aortic replacement permanently interrupts critical radicular arteries supplying the anterior spinal artery. To reduce the risk of spinal infarction during TAAA surgery, it is necessary to increase the ischemic tolerance of the spinal cord and maximize spinal cord perfusion. Typically, the anterior spinal artery is fed by several anterior radiculomedullary arteries, and, among these, the intercostal arteries arising from the thoracic and lumbar aorta are considered critical to spinal cord blood supply [15]. This is the rationale for the importance of critical segmental artery reimplantation in TAAA surgery. However, only a few of the segmental arteries supply radicular arteries contributing to 


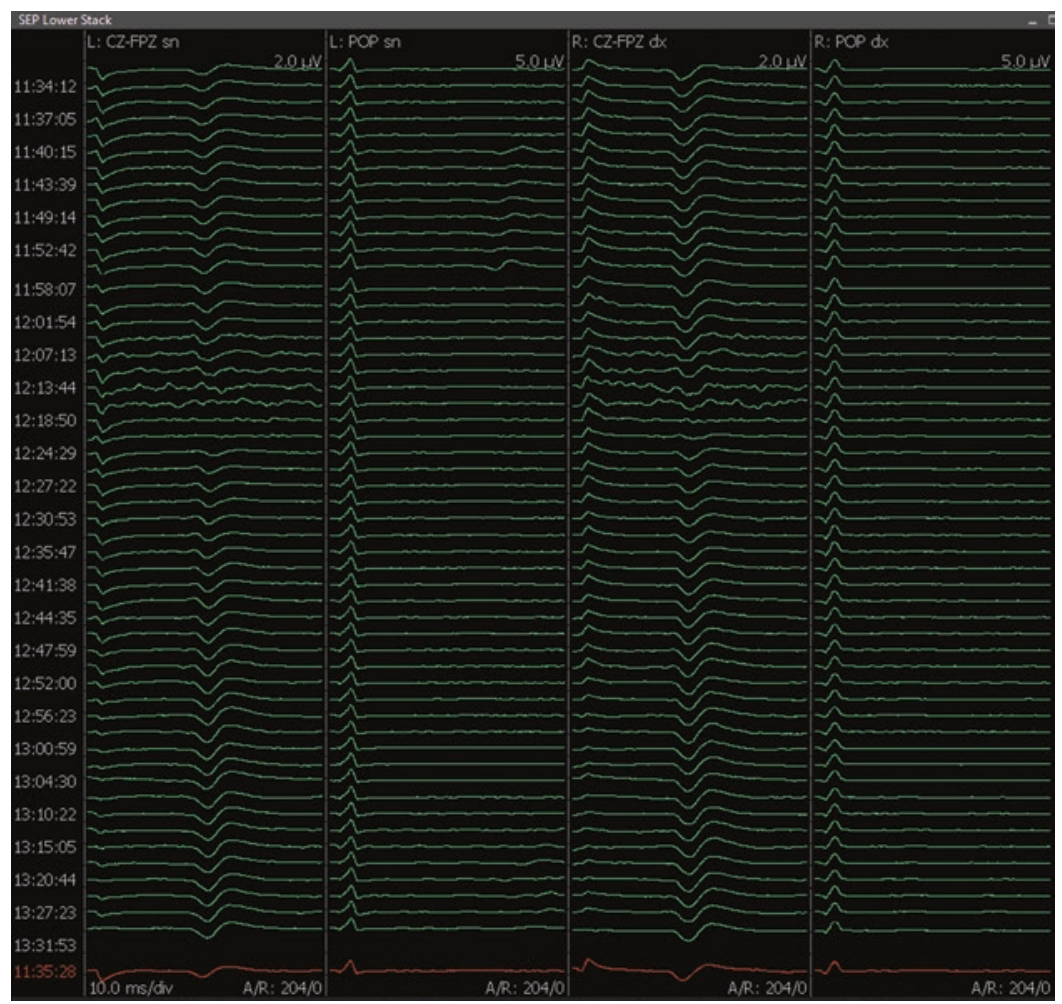

Fig. 26.3 Case 1: spinal cord ischemia after aorta crossclamping. In the figure, same patient (AM), of Fig. 26.2, $\mathrm{SSEP}$ time course is reported. From left to right: $\mathrm{CPz}-\mathrm{Fpz}$ derivation (first columns), popliteal fossa (second column) for left stimulation, $\mathrm{CPz}-\mathrm{Fpz}$ and popliteal fossa for right stimulation (third and fourth columns). Baseline tracings

the anterior spinal artery [16], and their identification could be difficult [17]. Furthermore, reimplanting intercostal arteries is technically challenging because open intercostals are often small and atherosclerotic in patients with TAAA [18]. The spinal cord has also a complex collateral circulation fed by other thoracic arteries that contribute significantly to anterior spinal cord blood flow $[16,19]$. The concept of collateral circulation explains how maintaining high arterial blood pressure reduces spinal cord ischemia and infarction in TAAA surgery.

Although majority of surgeons consider reimplantation of critical segmental arteries, controversies still exist about the clinical benefits [20, 21]. Several studies sought to prevent ischemia by reimplanting segmental arteries with particular focus on those arising between $\mathrm{T} 7$ and L1, (represented by the red line) are shown at the bottom of the stack. A brief amplitude decrease of cortical SSEPs was observed between 12:13 and 12:24, while no changes occurred in peripheral responses. However, the concomitant persistence of MEP changes motivated the intercostal arteries reimplantation

believing that paraplegia is the consequence of hypoperfusion after the sacrifice of critical spinal cord arteries [5, 22]. Because these reports combine intercostal reimplantation with other protective strategies, it is impossible to determine how much intercostal reattachment itself contributes to improve results. How many and which arteries have to be reimplanted is not completely clarified. This dilemma could be elucidated with preoperative radiological studies [23, 24], intraoperative neurophysiological monitoring $[25,26]$, or both $[27,28]$. SSEPs do not seem suitable for determination of critical segmental arteries because of a relative high false-positive rate [29]. More often the selective reconstruction of segmental arteries is guided by MEP monitoring $[30,31]$. According to this method, when the exclusion of an aortic segment results in ischemic 


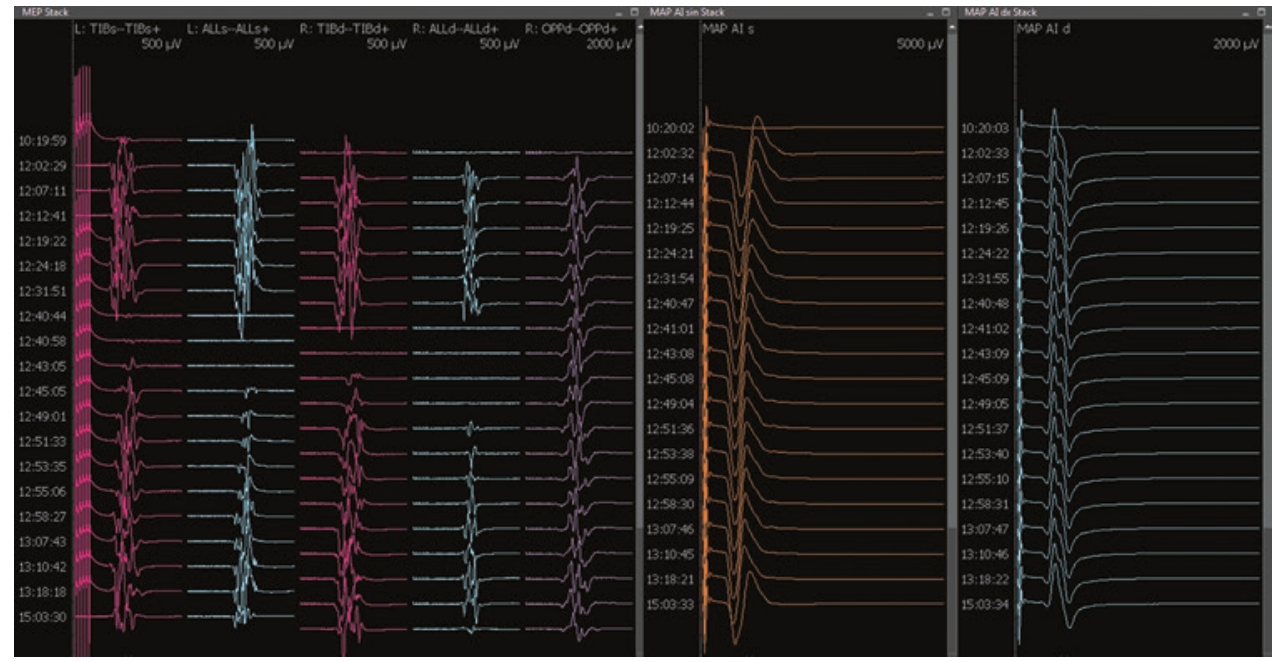

Fig. 26.4 Case 2: spinal cord ischemia related to intercostal artery blood reflux. MEP and CMAP time courses are reported for a patient suffering of TAAA type I (CA). From left to right: MEPs from the left tibialis anterior (first column), left abductor hallucis (second column), right tibialis anterior (third column), right abductor hallucis (fourth column), and right opponens pollicis (fifth column) and CMAPs from left and right abductor hallucis (sixth and seventh columns). Aorta cross-clamping occurs at 12:10. At 12:40 an amplitude reduction in MEPs is observed for muscles of the left and right inferior limb. No modification is observed for the upper limb, used as control. No modification is present for CMAPs. This situation, suggesting a central involvement, appears strictly related to a sudden, massive, blood reflux from intercostal arteries, occurring at 12:35. After intercostal sewing, MEPs rapidly come back to the baseline condition. No SSEP changes were observed: as known, the presence of synapses in the anterior horn gray matter makes MEPs more sensitive than SSEPs in detecting SCI

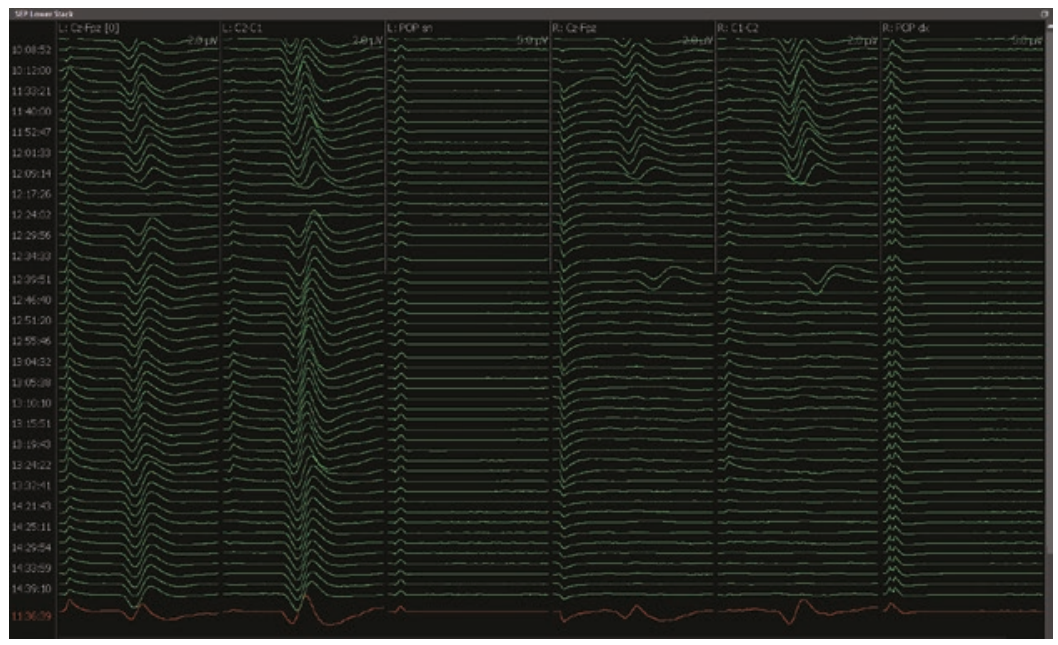

Fig. 26.5 Case 3: left hemispheric ischemic stroke. In the figure SSEP time courses for a patient suffering of TAAA type II (TV) are reported. From left to right: $\mathrm{CPz}-\mathrm{Fpz}$ derivation (first columns) and $\mathrm{C} 2-\mathrm{C} 1$ (second column), popliteal fossa (third column) for left stimulation and CPz-Fpz, $\mathrm{C} 1-\mathrm{C} 2$, and popliteal fossa for right stimulation (fourth, fifth, and sixth columns). Baseline tracings (represented by the red line) are shown at the bottom of the stack. Aorta cross-clamping occurs between 11:19 and 12:23. No MEP changes were observed throughout the surgery. A temporary amplitude decrease of cortical SSEPs and SAPs was observed between 12:09 and 12:29 in the left side and between 12:09 and 12:39 in the right side. After an initial recovery for left and right SSEPs, a sudden disappearance of right stimulus-related cortical SSEP derivation is observed, maintained until the end of the surgery. A CT scan after intervention showed a left hemispheric ischemic stroke. An involvement of the cortical territory of the middle cerebral artery (MCA) can explain a sudden disappearance of the cortical SSEP and the initial persistence of MEP response, because the transcranial electrical stimulation can depolarize pyramidal axons deeper in the cerebral hemisphere 


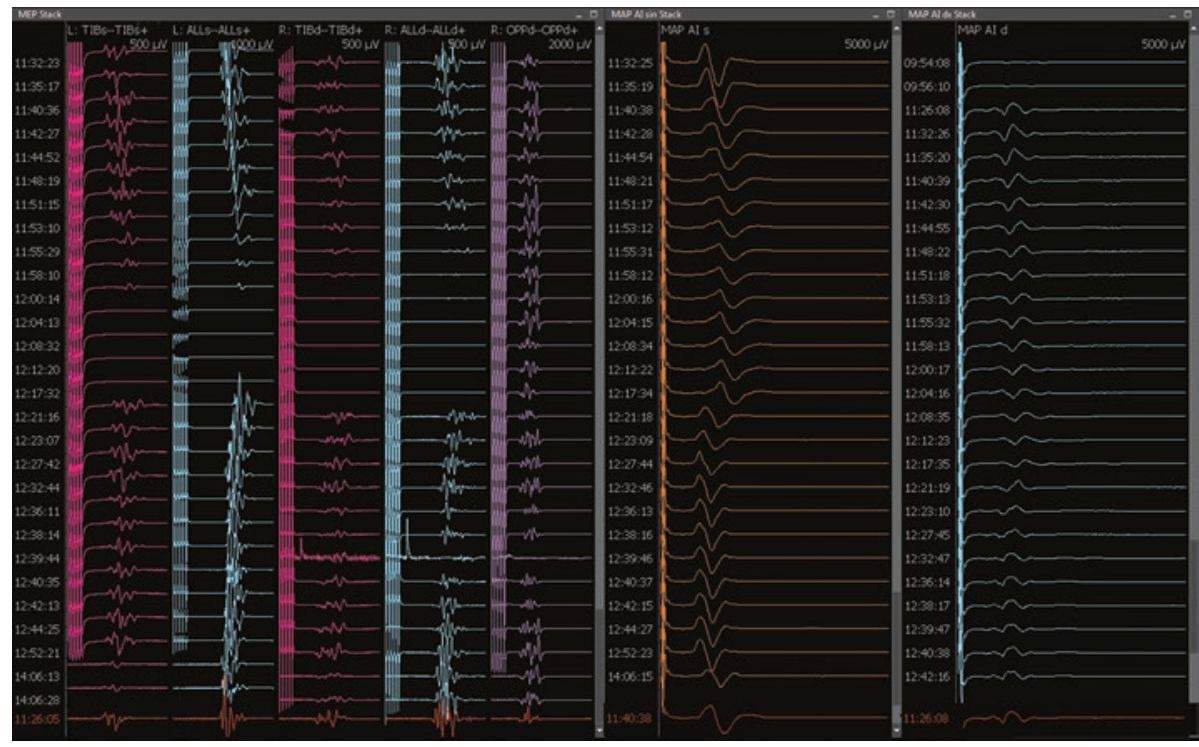

Fig. 26.6 Case 4: lower limb ischemia. MEP and CMAP time courses, for a patient (FL) suffering of TAAA type IV, are reported. No perfusion assistance is used during surgery. From left to right: MEPs from the left tibialis anterior (first column), left abductor hallucis (second column), right tibialis anterior (third column), right abductor hallucis (fourth column), and right opponens pollicis (fifth column) and CMAPs from the left and right abductor hallucis (sixth and seventh columns). Aorta cross-clamping occurs at 11:26. At 11:53 an amplitude reduction in MEPs is observed for muscles of the left and right inferior limbs. No modification is observed for the upper limb, used as control. A concomitant amplitude reduction and temporal spreading are shown by left and right CMAPs. This last modification is suggesting for a peripheral suffering. After aorta declamp, at 12:18, a quite rapid return to the baseline condition is achieved for both peripheral and central responses

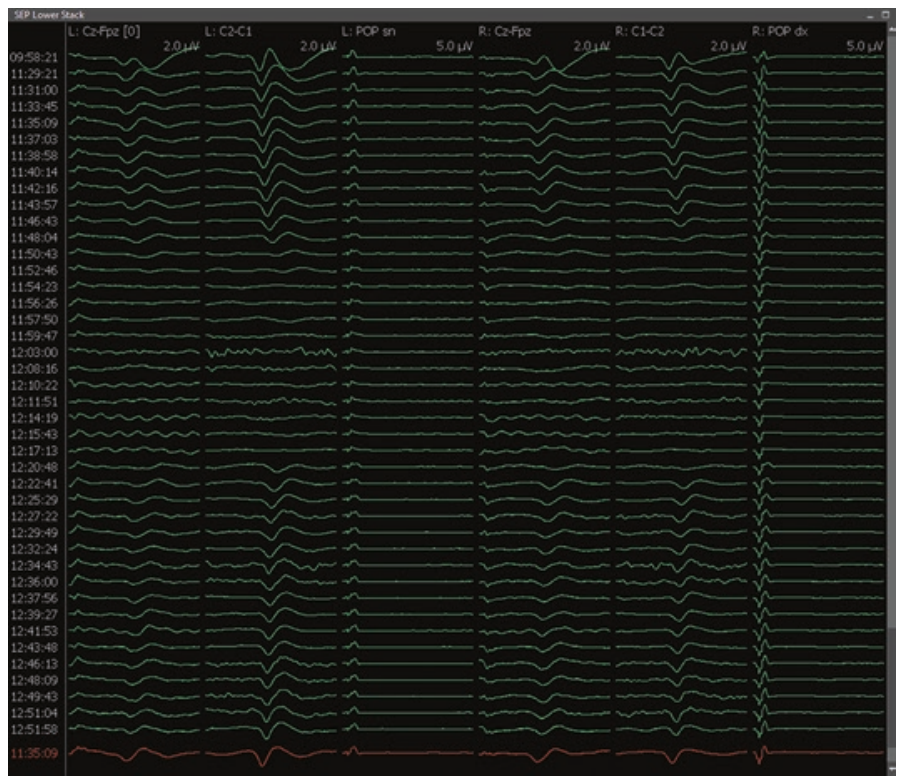

Fig. 26.7 Case 4: lower limb ischemia. For the same patient of Fig. 26.6 (FL), SSEP time course is reported. From left to right: $\mathrm{CPz}-\mathrm{Fpz}$ derivation (first columns), $\mathrm{C} 2-\mathrm{C} 1$ (second column), and popliteal fossa (third column) for left stimulation and $\mathrm{CPz}-\mathrm{Fpz}, \mathrm{C} 1-\mathrm{C} 2$, and popliteal fossa for right stimulation (fourth, fifth, and sixth columns). An amplitude decrease for scalp and peripheral derivations is observed between 11:46 and $12: 25$. Also in this case, a peripheral suffering is testified by an amplitude reduction, and temporal spreading of sensory action potential (SAP) registered at popliteal fossa as shown in columns 3 and 6 
MEP changes, the intercostal or lumbar arteries in that segment are considered critical to spinal cord blood supply and reimplanted.

The concept of selective reconstruction of critical arteries was developed by Svensson [32] in an attempt to avoid injury resulting from failure to restore spinal blood flow and to shorten the time required for implantation of the vessels, since reconstruction of the noncritical arteries is shown to be associated with increased risk of injury [33].

Other authors argue that reimplantation of the critical segmental arteries is unnecessary or not critical at least [20,21]. This idea is supported by the anatomic evidence that the Adamkiewicz artery (AKA) is not the single source of perfusion to the distal spinal cord $[34,35]$. Some of them reported a reduced paralysis risk without any intercostal reimplantation $[15,18,21]$, using other strategies to increase ischemic tolerance and maximize collateral circulation. However, other authors suggested that nonselective reimplantation of segmental arteries may be protective by increasing perfusion to the collateral network even if they are not the direct origin of the AKA [20,36]. Animal experiments show that when one intercostal artery is ligated, spinal cord blood flow arising from the preserved intercostals increases [37]. This suggests that any intercostal artery can supply blood to the spinal cord and become collateral circulation to the anterior spinal artery. In patients with reduced collateral circulation, reimplanting any intercostal in the critical zone from T8 to L1 may permanently increase perfusion pressure in the collateral network, and this may be the important factor in preventing spinal cord infarction. The advantages are the minimization of procedural time and the length of anastomotic line that carries a risk of bleeding. However, if the incidence of immediate neurological deficits is significantly reduced using both selective and empirical reimplantations, the incidence of delayed spinal cord ischemia is not completely clarified $[15,20,36]$.

Therefore, if MEP changes represent a warning toward the reimplantation of intercostal arteries, on the contrary, stable MEPs throughout the surgery could indicate that segmental artery reattachment could not be necessary [38].
In conclusion, literature data suggest that MEP monitoring is reliable in detecting SCI, allowing the determination of critical segmental arteries and the assessment of the effects of protective techniques. A multimodal IOM can be useful to assess the various factors that may contribute to SCI, to help guiding specific measures to reduce neural risk and minimize the overall ischemic time by focusing efforts only on the necessary maneuvers.

\section{References}

1. Melissano G, Bertoglio L, Mascia D, Rinaldi E, Del Carro U, Nardelli P, et al. Spinal cord ischemia is multifactorial: what is the best protocol? J Cardiovasc Surg. 2016;57(2):191-201.

2. Kahn RA, Stone ME, Moskowitz DM. Anesthetic consideration for descending thoracic aortic aneurysm repair. Semin Cardiothorac Vasc Anesth. 2007;11(3):205-23.

3. Greenberg RK, Lytle B. Endovascular repair of thoracoabdominal aneurysms. Circulation. 2008;117(17):2288-96.

4. Cambria RP, Davison JK, Carter C, Brewster DC, Chang Y, Clark KA, et al. Epidural cooling for spinal cord protection during thoracoabdominal aneurysm repair: a five-year experience. J Vasc Surg. 2000;31(6):1093-102.

5. Coselli JS, Bozinovski J, LeMaire SA. Open surgical repair of 2286 thoracoabdominal aortic aneurysms. Ann Thorac Surg. 2007;83(2):S862-4. discussion S890-892

6. Kieffer E, Chiche L, Godet G, Koskas F, Bahnini A, Bertrand M, et al. Type IV thoracoabdominal aneurysm repair: predictors of postoperative mortality, spinal cord injury, and acute intestinal ischemia. Ann Vasc Surg. 2008;22(6):822-8.

7. Bicknell CD, Cowan AR, Kerle MI, Mansfield AO, Cheshire NJW, Wolfe JHN. Renal dysfunction and prolonged visceral ischaemia increase mortality rate after suprarenal aneurysm repair. Br J Surg. 2003;90(9):1142-6.

8. Hiratzka LF, Bakris GL, Beckman JA, Bersin RM, Carr VF, Casey DE, et al. 2010 ACCF/AHA/AATS/ ACR/ASA/SCA/SCAI/SIR/STS/SVM guidelines for the diagnosis and management of patients with thoracic aortic disease: executive summary. A report of the American College of Cardiology Foundation/American Heart Association Task Force on Practice Guidelines, American Association for Thoracic Surgery, American College of Radiology, American Stroke Association, Society of Cardiovascular Anesthesiologists, Society for Cardiovascular Angiography and Interventions, Society of Interventional Radiology, Society of 
Thoracic Surgeons, and Society for Vascular Medicine. Catheter Cardiovasc Interv Off J Soc Card Angiogr Interv. 2010;76(2):E43-86.

9. Sloan TB, Edmonds HL, Koht A. Intraoperative electrophysiologic monitoring in aortic surgery. J Cardiothorac Vasc Anesth. 2013;27(6):1364-73.

10. Nielsen VK, Kardel T. Temporospatial effects on orthodromic sensory potential propagation during ischemia. Ann Neurol. 1981;9(6):597-604.

11. Tanaka Y, Kawaguchi M, Noguchi Y, Yoshitani K, Kawamata M, Masui K, et al. Systematic review of motor evoked potentials monitoring during thoracic and thoracoabdominal aortic aneurysm open repair surgery: a diagnostic meta-analysis. J Anesth. 2016;30(6):1037-50.

12. Svensson LG, Rickards E, Coull A, Rogers G, Fimmel CJ, Hinder RA. Relationship of spinal cord blood flow to vascular anatomy during thoracic aortic cross-clamping and shunting. J Thorac Cardiovasc Surg. 1986;91(1):71-8.

13. Marini CP, Levison J, Caliendo F, Nathan IM, Cohen JR. Control of proximal hypertension during aortic cross-clamping: its effect on cerebrospinal fluid dynamics and spinal cord perfusion pressure. Semin Thorac Cardiovasc Surg. 1998;10(1):51-6.

14. Marsala M, Sorkin LS, Yaksh TL. Transient spinal ischemia in rat: characterization of spinal cord blood flow, extracellular amino acid release, and concurrent histopathological damage. J Cereb Blood Flow Metab Off J Int Soc Cereb Blood Flow Metab. 1994;14(4):604-14.

15. Acher CW, Wynn MM, Hoch JR, Popic P, Archibald J, Turnipseed WD. Combined use of cerebral spinal fluid drainage and naloxone reduces the risk of paraplegia in thoracoabdominal aneurysm repair. J Vasc Surg. 1994;19(2):236-46. discussion 247-248

16. Christiansson L, Ulus AT, Hellberg A, Bergqvist D, Wiklund L, Karacagil S. Aspects of the spinal cord circulation as assessed by intrathecal oxygen tension monitoring during various arterial interruptions in the pig. J Thorac Cardiovasc Surg. 2001;121(4):762-72.

17. Williams GM, Roseborough GS, Webb TH, Perler BA, Krosnick T. Preoperative selective intercostal angiography in patients undergoing thoracoabdominal aneurysm repair. J Vasc Surg. 2004;39(2):314-21.

18. Griepp RB, Ergin MA, Galla JD, Lansman S, Khan $\mathrm{N}$, Quintana C, et al. Looking for the artery of Adamkiewicz: a quest to minimize paraplegia after operations for aneurysms of the descending thoracic and thoracoabdominal aorta. J Thorac Cardiovasc Surg. 1996;112(5):1202-13. discussion 1213-1215

19. Lazorthes G, Gouaze A, Zadeh JO, Santini JJ, Lazorthes Y, Burdin P. Arterial vascularization of the spinal cord. Recent studies of the anastomotic substitution pathways. J Neurosurg. 1971;35(3):253-62.

20. Acher CW, Wynn MM, Mell MW, Tefera G, Hoch JR. A quantitative assessment of the impact of intercostal artery reimplantation on paralysis risk in thoracoabdominal aortic aneurysm repair. Ann Surg. 2008;248(4):529-40.
21. Etz CD, Halstead JC, Spielvogel D, Shahani R, Lazala R, Homann TM, et al. Thoracic and thoracoabdominal aneurysm repair: is reimplantation of spinal cord arteries a waste of time? Ann Thorac Surg. 2006;82(5):1670-7.

22. Cambria RP, Clouse WD, Davison JK, Dunn PF, Corey M, Dorer D. Thoracoabdominal aneurysm repair: results with 337 operations performed over a 15-year interval. Ann Surg. 2002;236(4):471-9. discussion 479

23. Yamada N, Takamiya M, Kuribayashi S, Okita Y, Minatoya K, Tanaka R. MRA of the Adamkiewicz artery: a preoperative study for thoracic aortic aneurysm. J Comput Assist Tomogr. 2000;24(3):362-8.

24. Kawaharada N, Morishita K, Fukada J, Yamada A, Muraki S, Hyodoh $\mathrm{H}$, et al. Thoracoabdominal or descending aortic aneurysm repair after preoperative demonstration of the Adamkiewicz artery by magnetic resonance angiography. Eur J Cardio-Thorac Surg Off J Eur Assoc Cardio-Thorac Surg. 2002;21(6): 970-4.

25. Jacobs MJ, de Mol BA, Elenbaas T, Mess WH, Kalkman CJ, Schurink GW, et al. Spinal cord blood supply in patients with thoracoabdominal aortic aneurysms. J Vasc Surg. 2002;35(1):30-7.

26. van Dongen EP, Schepens MA, Morshuis WJ, ter Beek HT, Aarts LP, de Boer A, et al. Thoracic and thoracoabdominal aortic aneurysm repair: use of evoked potential monitoring in 118 patients. J Vasc Surg. 2001;34(6):1035-40.

27. Ogino H, Sasaki H, Minatoya K, Matsuda H, Yamada N, Kitamura S. Combined use of adamkiewicz artery demonstration and motor-evoked potentials in descending and thoracoabdominal repair. Ann Thorac Surg. 2006;82(2):592-6.

28. Takahashi S, Orihashi K, Imai K, Mizukami T, Takasaki T, Sueda T. Cold blood spinoplegia under motor-evoked potential monitoring during thoracic aortic surgery. J Thorac Cardiovasc Surg. 2011;141(3):755-61.

29. Crawford ES, Mizrahi EM, Hess KR, Coselli JS, Safi HJ, Patel VM. The impact of distal aortic perfusion and somatosensory evoked potential monitoring on prevention of paraplegia after aortic aneurysm operation. J Thorac Cardiovasc Surg. 1988;95(3):357-67.

30. Shiiya N, Yasuda K, Matsui Y, Sakuma M, Sasaki S. Spinal cord protection during thoracoabdominal aortic aneurysm repair: results of selective reconstruction of the critical segmental arteries guided by evoked spinal cord potential monitoring. J Vasc Surg. 1995;21(6):970-5.

31. Jacobs MJ, Meylaerts SA, de Haan P, de Mol BA, Kalkman CJ. Strategies to prevent neurologic deficit based on motor-evoked potentials in type I and II thoracoabdominal aortic aneurysm repair. J Vasc Surg. 1999;29(1):48-57. discussion 57-59

32. Svensson LG, Patel V, Robinson MF, Ueda T, Roehm JO, Crawford ES. Influence of preservation or perfusion of intraoperatively identified spinal cord blood supply on spinal motor evoked potentials 
and paraplegia after aortic surgery. J Vasc Surg. 1991;13(3):355-65.

33. Svensson LG, Hess KR, Coselli JS, Safi HJ. Influence of segmental arteries, extent, and atriofemoral bypass on postoperative paraplegia after thoracoabdominal aortic operations. J Vasc Surg. 1994;20(2): 255-62.

34. Etz CD, Kari FA, Mueller CS, Silovitz D, Brenner RM, Lin H-M, et al. The collateral network concept: a reassessment of the anatomy of spinal cord perfusion. J Thorac Cardiovasc Surg. 2011;141(4):1020-8.

35. Biglioli P, Roberto M, Cannata A, Parolari A, Fumero A, Grillo F, et al. Upper and lower spinal cord blood supply: the continuity of the anterior spinal artery and the relevance of the lumbar arteries. $\mathrm{J}$ Thorac Cardiovasc Surg. 2004;127(4):1188-92.

36. Park KH, Lim C, Kim TH, Park I, Jung Y. Outcome of extensive descending aorta repair adopting present concepts of spinal cord preservation. J Cardiovasc Surg. 2016;57(1):58-65.

37. Uezu T, Koja K, Kuniyoshi Y, Miyagi K, Shimoji M, Arakaki K, et al. Blood distribution to the anterior spinal artery from each segment of intercostal and lumbar arteries. J Cardiovasc Surg. 2003;44(5):637-45.

38. Conrad MF, Ergul EA, Patel VI, Cambria MR, Lamuraglia GM, Simon M, et al. Evolution of operative strategies in open thoracoabdominal aneurysm repair. J Vasc Surg. 2011;53(5):1195-1201.e1. 